\title{
A comparative study of the long-term light variations of six young irregular variables
}

\author{
J. Gürtler ${ }^{1}$, C. Friedemann ${ }^{1}$, H.-G. Reimann ${ }^{1}$, E. Splittgerber ${ }^{2}$, and E. Rudolph ${ }^{3}$ \\ 1 Friedrich-Schiller-Universität Jena, Astrophysikalisches Institut und Universitäts-Sternwarte, Schillergäßchen 2, D-07745 \\ Jena, Germany \\ 2 Ludwig-Stur-Straße 5, D-06108 Halle/Saale, Germany \\ 3 Lieselotte-Hermann-Straße 38a, D-07747 Jena, Germany
}

Received April 17, 1998; accepted September 13, 1999

\begin{abstract}
We investigated the long-term light variations of six young irregular variables, all of them UX Orionis stars or related objects. For this aim we determined new photographic magnitudes for the five stars VX Cas, BH Cep, BO Cep, SV Cep, and RZ Psc and made use of similar data for WW Vul published independently. The magnitudes were derived from Argelander brightness estimates performed on plates of the Harvard College Observatory and Sonneberg Observatory plate collections. The data cover a period of about 100 years and are available at the Centre des Données Stellaires, Strasbourg. The new data were supplemented by multicolour photoelectric measurements taken from the literature and visual estimates collected by the AAVSO. The resulting lightcurves are displayed and discussed. Algol-like minima are observed with all the stars. Their properties are discussed under the presumption that they are caused by circumstellar dust clouds orbiting the stars. Parameters of the circumstellar dust shells are derived from modelling the observed infrared excess radiation and compared with constraints from the light variations.
\end{abstract}

Key words: stars: individual: VX Cas, BH Cep, BO Cep, SV Cep, RZ Psc, WW Vul - stars: variables: general circumstellar matter

\section{Introduction}

The circumstellar dust shells around young stars have attracted much attention in recent years. A particularly interesting group is the so-called UX Orionis stars. In addition to other components, the light variations of

Send offprint requests to: J. Gürtler

e-mail: guertler@astro.uni-jena.de these variables show short-lived minima which remind of the lightcurves of Algol stars. Following a suggestion by Wenzel (1969), these minima are widely attributed to the occultation of the stars by circumstellar dust clouds. While the infrared excesses reveal the presence of circumstellar dust in most cases, the Algol-like light variations are strong evidence for the shells being dominated by an ensemble of individual dust clouds rather than characterized by a more or less smooth distribution of the circumstellar matter.

The light variations due to varying circumstellar extinction open a unique way to study the structure of the circumstellar dust shells, the properties of the individual dust clouds, and the characteristics of the dust grains (see, e.g., Friedemann et al. 1994a, 1995; Reimann et al. 1997). For instance, the discussion of multicolour photoelectric data of individual Algol-like minima may provide information on the optical properties of the dust grains in individual circumstellar clouds (e.g., through the reddening law). However, only the study of the long-term behaviour of the light variations provides information on the statistical properties of the cloud ensemble and the structure of the circumstellar envelope. For this aim, we have launched a programme to investigate the long-term behaviour of selected UX Ori stars.

Since there has been no dedicated monitoring of UX Ori stars, the only way to get complete lightcurves over a long interval of time is to make use of photographic plate collections. The richest plate collections are located at the Harvard College Observatory and at Sonneberg Observatory. Most of their stocks is due to the systematic sky patrol work that was done at Harvard from 1898 to 1989 with a break from 1954 to 1962 and that has been done at Sonneberg since 1926 with only a short break in 1995. Thus, the archives of both observatories complement each other and exploiting both of them provides data for a time interval of nearly 100 years. 
The aim of the present paper is twofold. First, we will report the results of our brightness estimates on photographic sky patrol plates in the archives of Harvard College Observatory for VX Cas, BH Cep, BO Cep, SV Cep, and RZ Psc and of Sonneberg Observatory for VX Cas, BH Cep, BO Cep, and SV Cep. In the case of RZ Psc the photographic data obtained from the Sonneberg Observatory plates were already published by Home et al. (1994). Second, we wish to present a comparative study of the light variations of these stars with particular emphasis on the Algol-like minima. We will include the UX Ori star WW Vul, for which we have already presented a similar data set elsewhere (Friedemann et al. 1994b, 1996).

\section{The star sample}

In Table 1 we summarize relevant data for the stars studied here. Originally, these stars were taken from the Third Catalog of Emission-line Stars of the Orion Population by Herbig \& Bell (1988) by requiring that they should show photoelectrically observed Algol-like minima. Accepting the suggestion by Wenzel (1969) that these Algol-like minima originate from occultations of the stars by circumstellar dust clouds, we have also required that the stars should show an excess of near-infrared radiation as evidence of thermal emission from circumstellar dust. An additional constraint dictated by practical considerations was that the apparent magnitude of the stars had to be brighter than the plate limits during their minima.

Although RZ Psc is neither contained in Herbig \& Bell's catalogue nor an IRAS point source, it was included in our study because it frequently shows deep Algol-like minima of the UX Ori type (see, e.g., Zaytseva 1985). It deserves particular interest because of its late spectral type. The non-detection by IRAS does not argue against the presence of an circumstellar dust shell because the emission of a dust shell similar to those around WW Vul and SV Cep is well below the detection limit of IRAS (Friedemann et al. 1994a).

\section{New photographic magnitudes from Harvard and Sonneberg sky patrol plates}

\subsection{Comparison star sequences}

For our aim to determine the long-term behaviour of the light variations, brightness estimates by eye are the only appropriate way to cope with the large numbers of plates. The accuracy is expected to be sufficiently high to detect Algol-like minima with amplitudes $\geq 0.5 \mathrm{mag}$. To have a reliable basis for the brightness estimates and to limit the photometric errors, we determined the magnitudes of a large number of comparison stars. The necessary observations were done with a CCD camera attached to the $60 / 90 / 180 \mathrm{~cm}$ Schmidt telescope of the UniversitätsSternwarte Jena at Großschwabhausen Observing Station. As primary standards we used the stars observed by Pugach \& Kovalchuk (1983). The only omission was their comparison star "g" for BH Cep. This star is very red and the authors consider their magnitude measurement very uncertain. Therefore we discarded this star as second standard star for the photometric sequence for BH Cep and used comparison star " $\alpha$ " from Wenzel \& Brückner (1978) instead. Reduction of our photometric data gives $V=11$ m 66 for Pugach \& Kovalchuk's star "g". (The very same star is denoted by "i" in Table 2.) The colour system of our CCD camera is virtually identical with the standard $U B V$ system. Therefore, no colour transformations have been applied.

Typical values for the rms errors of the magnitudes derived by our CCD photometry are $\Delta m \approx \pm 0.02 \mathrm{mag}$ for $9^{\mathrm{m}} \lesssim B \lesssim 12^{\mathrm{m}}, \Delta m \approx \pm 0.03$ mag for $13^{\mathrm{m}} \lesssim B \lesssim 14^{\mathrm{m}}$, and $\Delta m \approx \pm 0.10 \mathrm{mag}$ for $14^{\mathrm{m}} \lesssim B \lesssim 14.5^{\mathrm{m}}$.

In Fig. 1 the comparison stars for VX Cas, BH Cep, BO Cep, and SV Cep are identified. In the case of SV Cep we present an updated version of our earlier identification map (Friedemann et al. 1992). To avoid confusion, we left the labelling of the comparison stars unchanged but added two more comparison stars. Table 2 gives the $B$ and $V$ magnitudes measured by us. Since the $B$ magnitudes are very similar to photographic (blue) magnitudes, they were used with the visual estimates on the photographic plates without any further transformations.

For an identification map of the comparison stars for the variable RZ Psc and their magnitudes see Friedemann et al. (1995).

\subsection{Accuracy of the magnitude estimates}

Within the long time interval covered by our work, many different sorts of photographic emulsions were used in the sky patrol work. In order to cope with this diversity, we restricted ourselves to blue sensitive photographic plates. Systematic differences were minimized by using the same sequences of comparison stars on all plates. The accuracy of the brightness estimates depends on the local sensitivity fluctuations of the emulsions and the accuracy of the estimates themselves. The individual step sizes among the authors varied from 0.08 up to 0.15 mag. Thus, the uncertainty of the magnitudes should not exceed $\Delta m \approx \pm 0.3 \mathrm{mag}$ in most cases. This is in accordance with the statistical error which we derived when several plates per night were available.

A feature common to all our data is the marked horizontal striation in the lightcurves (see Fig. 2). This striation grows to prominent voids in some regions of the lightcurves of WW Vul, BH Cep, and SV Cep. The fine striation is a natural consequence of the fact that due to the limited accuracy of the brightness estimation by eye 


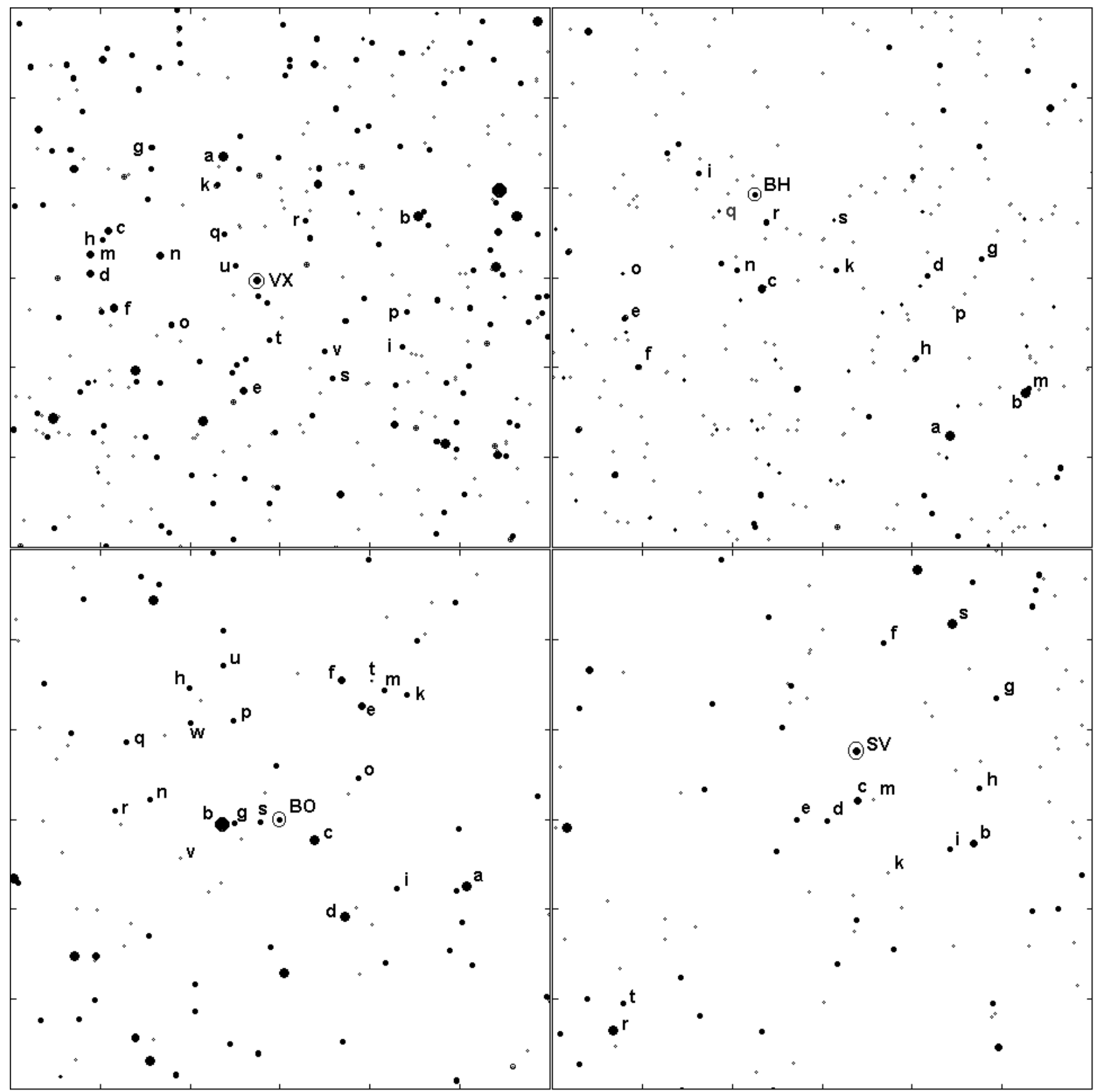

Fig. 1. Identification maps for the comparison stars for VX Cas, BH Cep, BO Cep, and SV Cep adopted from the GSC (2000). North is on the top and east to the left. The distance between tic marks on both axes corresponds to $5^{\prime}$ on the sky. The J2000.0 coordinates of the centres of the fields are $\alpha=00^{\mathrm{h}} 31^{\mathrm{m}} 20^{\mathrm{s}}$ and $\delta=61^{\circ} 59^{\prime} 00^{\prime \prime}$ for VX Cas (upper left); $\alpha=22^{\mathrm{h}} 01^{\mathrm{m}} 00^{\mathrm{s}}$ and $\delta=69^{\circ} 40^{\prime} 00^{\prime \prime}$ for BH Cep (upper right); $\alpha=22^{\mathrm{h}} 16^{\mathrm{m}} 54^{\mathrm{s}}$ and $\delta=70^{\circ} 03^{\prime} 49^{\prime \prime}$ for BO Cep (lower left); $\alpha=22^{\mathrm{h}} 22^{\mathrm{m}} 00^{\mathrm{s}}$ and $\delta=73^{\circ} 36^{\prime} 40^{\prime \prime}$ for SV Cep (lower right)

the magnitudes are assigned in discrete steps to the variable. The broader horizontal voids of missing data points have an additional cause. As already discussed with the presentation of the data for WW Vul (Friedemann et al. 1994b), statistical analyses revealed that some observers were evidently reluctant to establish equal brightness for the variable and the comparison star. As a consequence data points which represent the magnitudes of the comparison stars are small in number or absent at all. The vertical width of the voids does not exceed 2 steps and, therefore, the maximum error estimated in the last paragraph is not increased by this personal bias.

No systematic differences were found among the brightness estimates by different observers in overlapping 
Table 1. Fundamental data of the sample stars

\begin{tabular}{ccccccc}
\hline Star & $\begin{array}{c}\alpha_{1950} \\
\left({ }^{\mathrm{m} \mathrm{m} \mathrm{s}}\right)\end{array}$ & $\begin{array}{c}\delta_{1950} \\
\left({ }^{\circ}{ }^{\prime \prime}\right)\end{array}$ & IRAS & Spectral type & $\begin{array}{c}\text { Type of } \\
\text { variability }\end{array}$ & $\begin{array}{c}\text { Amplitude } \\
(\mathrm{mag})\end{array}$ \\
\hline VX Cas & 002840.4 & 614117.3 & $00286+6142$ & A0/3e & Isa & $10.5-13.3(V)$ \\
RZ Psc & 010656.0 & 274130 & - & K0IV & Isb: & $11.3-13.8(V)$ \\
WW Vul & 192350 & +210630 & $19238+2106$ & A0V & Isa & $10.25-12.94(V)$ \\
BH Cep & 220038.2 & 693006.3 & $22006+6930$ & A/F5Ve $\alpha$ & Isb & $10.8-12.7(V)$ \\
BO Cep & 221540.2 & 694844.4 & $22156+6948$ & F2e $\alpha$ & Insb & $12.0-13.7(B)$ \\
SV Cep & 222034.0 & 732516.9 & $22205+7325$ & A0e $\alpha$ & Isa & $10.35-12.15(V)$ \\
\hline
\end{tabular}

References: Kholopov (1985); Thé et al. (1994).

Table 2. $B$ and $V$ magnitudes of the comparison stars

\begin{tabular}{|c|c|c|c|c|c|c|c|c|c|c|c|}
\hline \multicolumn{3}{|c|}{ VX Cas } & \multicolumn{3}{|c|}{ ВН Cep } & \multicolumn{3}{|c|}{ BO Cep } & \multicolumn{3}{|c|}{ SV Cep } \\
\hline Star & $\bar{B}$ & $\bar{V}$ & Star & $\bar{B}$ & $\bar{V}$ & Star & $\bar{B}$ & $\bar{V}$ & Star & $\bar{B}$ & $\bar{V}$ \\
\hline $\mathrm{a}$ & 10.38 & 9.31 & $\mathrm{a}$ & 9.95 & - & $\mathrm{a}$ & 8.87 & - & $\mathrm{r}$ & 10.11 & 9.74 \\
\hline b & 10.57 & 9.63 & $\mathrm{~b}$ & 10.52 & 10.12 & b & 9.19 & - & $\mathrm{s}$ & 10.76 & 10.34 \\
\hline $\mathrm{c}$ & 11.31 & 11.03 & $\mathrm{c}$ & 11.40 & 11.08 & $\mathrm{c}^{a}$ & 11.60 & 10.94 & $\mathrm{~b}^{b}$ & 10.94 & 10.73 \\
\hline d & 11.42 & 11.03 & d & 11.66 & 11.11 & d & 11.68 & 10.10 & $c$ & 11.54 & 11.10 \\
\hline e & 11.44 & 11.21 & e & 12.12 & 11.52 & e & 12.03 & 11.44 & $\mathrm{t}$ & 11.90 & 11.66 \\
\hline $\mathrm{f}$ & 11.80 & 10.83 & f & 12.16 & 11.59 & f & 12.14 & 10.83 & $\mathrm{f}$ & 12.55 & 12.17 \\
\hline $\mathrm{g}$ & 11.81 & 11.55 & $\mathrm{~g}$ & 12.58 & 11.93 & $\mathrm{~g}$ & 12.63 & 12.06 & $\mathrm{e}$ & 12.80 & 11.66 \\
\hline $\mathrm{h}$ & 12.13 & 11.48 & h & 12.77 & 11.91 & h & 12.83 & 11.66 & $\mathrm{~g}$ & 12.82 & 12.36 \\
\hline $\mathrm{i}$ & 12.22 & 11.43 & i & 13.08 & 11.66 & i & 12.85 & 12.14 & i & 13.05 & 12.54 \\
\hline $\mathrm{k}$ & 12.67 & 12.26 & $\mathrm{k}$ & 13.42 & 12.27 & $\mathrm{k}$ & 12.88 & 11.84 & d & 13.28 & 12.77 \\
\hline $\mathrm{m}$ & 12.71 & 10.93 & $\mathrm{~m}$ & 13.65 & 12.84 & $\mathrm{~m}$ & 13.23 & 12.80 & $\mathrm{~h}$ & 13.58 & 12.63 \\
\hline $\mathrm{n}$ & 12.74 & 11.39 & $\mathrm{n}$ & 13.87 & 13.11 & $\mathrm{n}$ & 13.39 & 11.67 & $\mathrm{k}$ & 13.88 & 13.30 \\
\hline o & 12.75 & 12.00 & O & 13.95 & 13.23 & O & 13.42 & 12.74 & $\mathrm{~m}$ & 14.48 & 13.96 \\
\hline $\mathrm{p}$ & 12.96 & 12.54 & $\mathrm{p}$ & 14.20 & 13.27 & $\mathrm{p}$ & 13.44 & 12.20 & & & \\
\hline$q$ & 13.20 & 12.81 & $q$ & 14.26 & 13.39 & $q$ & 13.78 & 12.99 & & & \\
\hline $\mathrm{r}$ & 13.25 & 12.45 & $\mathrm{r}$ & 14.34 & 12.64 & $\mathrm{r}$ & 13.90 & 12.67 & & & \\
\hline $\mathrm{s}$ & 13.56 & 12.92 & $\mathrm{~s}$ & 14.54 & 13.69 & $\mathrm{~s}$ & 14.04 & 12.97 & & & \\
\hline $\mathrm{t}$ & 13.58 & 12.85 & & & & $\mathrm{t}$ & 14.26 & 13.62 & & & \\
\hline $\mathrm{u}$ & 13.70 & 12.41 & & & & $\mathrm{u}$ & 14.27 & 12.94 & & & \\
\hline $\mathrm{v}$ & 14.17 & 13.26 & & & & $\mathrm{v}$ & 14.33 & 13.61 & & & \\
\hline & & & & & & $\mathrm{w}$ & 14.52 & 13.26 & & & \\
\hline & & & & & & $\mathrm{x}$ & 14.62 & 13.03 & & & \\
\hline
\end{tabular}

${ }^{a}$ Close double star.

${ }^{b}$ Possibly variable. The star brightened up sporadically by about 0.5 mag.

regions of the lightcurves. There are also no systematic differences between the eye estimates and the photoelectric measurements, as the inclusion of the latter in Fig. 2 shows. Of course, we must keep in mind that the accuracy of the magnitudes derived by visual brightness estimates is very low if they are compared with photoelectric data. Nevertheless, brightness estimates remain the only effective way to provide data for statistical analyses of lightcurves as long as the information of the plates is not yet available in digitized form.

\subsection{The new data}

Table 3 provides basic information on the collected data, such as the time intervals covered, the number of useful plates, and the name of the person who did the estimates.
The magnitudes, which cover the period from 1898 to 1997 in most cases, are available in electronic form from the Centre des Données Stellaires at Strasbourg, France.

\section{Discussion of the light variations}

\subsection{The available photometric data}

In addition to the new data presented in the foregoing section, our study of the light variations of six UX Ori stars includes supplementary observational data from various sources. A short overview of these data follows.

$S V$ Cep: The results of a search of the literature for photometric data were published by Friedemann et al. (1992). In the meantime the results of visual brightness estimates collected by the AAVSO were made available to 
Table 3. Basic information on the new data sets

\begin{tabular}{|c|c|c|c|c|c|c|c|}
\hline Star & $\begin{array}{c}\text { Period } \\
(\mathrm{JD})\end{array}$ & $\begin{array}{r}B_{\max } \\
\left({ }^{\mathrm{m}}\right) \\
\end{array}$ & $\begin{array}{r}B_{\min } \\
\left({ }^{\mathrm{m}}\right)\end{array}$ & Archive & $N$ & $\bar{\Sigma}$ & Authors \\
\hline \multirow[t]{2}{*}{ VX Cas } & $2414633-2439917$ & 10.5 & 12.6 & Harvard & 1817 & & Gü \\
\hline & $2436426-2450370$ & & & Sonneberg & 1020 & 2837 & $\mathrm{Ru}$ \\
\hline \multirow[t]{2}{*}{ RZ Psc } & $2414631-2439141$ & 10.9 & 13.8 & Harvard & 964 & & Rei \\
\hline & $2425510-2448983$ & & & Sonneberg & $1022^{a}$ & 1986 & Home \\
\hline \multirow{2}{*}{ BH Cep } & $2414673-2439692$ & 11.2 & 12.2 & Harvard & 2018 & & Fr \\
\hline & $2436019-2450463$ & & & Sonneberg & 1958 & 3976 & Spl \\
\hline \multirow[t]{2}{*}{ BO Сер } & $2414673-2439692$ & 11.7 & 13.3 & Harvard & 1702 & & $\mathrm{Fr}$ \\
\hline & $2436019-2450463$ & & & Sonneberg & 1977 & 3679 & Spl \\
\hline \multirow[t]{2}{*}{ SV Cep } & $2414673-2447857$ & 9.7 & 12.7 & Harvard & 2232 & & Fr, Rei \\
\hline & $2425503-2450463$ & & & Sonneberg & 2298 & 4530 & Fr, Gü, Spl \\
\hline
\end{tabular}

${ }^{a}$ Most of these data have been already published by Home et al. (1994).

us (Mattei 1996, private communication). A small number of photoelectric uvby observations were reported by Reimann \& Friedemann (1991). Further UBV data were published by Kardopolov \& Filip'ev (1981).

$W W$ Vul: Visual brightness estimates on sky patrol plates from the archives of Sonneberg, Bamberg, and Harvard observatories were published by Friedemann et al. (1994b, 1996). The results of a literature search are given by Friedemann et al. (1993). In the present study we include new $U B V$ observations by Zaytseva \& Lyutyi (1997) and visual estimates provided by the AAVSO (Mattei 1996, private communication).

RZ Psc: Brightness estimates based on the plates in the Sonneberg Observatory Archive were published by Home et al. (1994). Photoelectric $U B V R$ data were given by Kardopolov et al. (1980) and Zaytseva (1985). Wenzel (1991) reported a number of photoelectric $U B V$ observations.

BH Cep: Photoelectric UBV observations were reported by Zhelezniakova \& Kardopolov (1980).

BO Cep: Kardopolov \& Shutiomova (1980) gave photoelectric $U B V$ observations.

$V X$ Cas: Photoelectric $U B V R$ and $U B V$ measurements were published by Kardopolov \& Filip'ev (1981, 1985a,b), Pugach (1979), and Zaytseva (1970), resp. Additional $U B V R$ data were communicated to us by Shevchenko (private communication).

\subsection{The long-term lightcurves}

Figure 2 presents plots of all the photometric data that have been available to us. Here and in the following we will generally use $B$ magnitudes and transform the brightness data to this system if necessary.

The lightcurves of the six stars appear quite different. While the lightcurves of SV Cep and WW Vul show conspicuous wave-like patterns with amplitudes of up to $1 \mathrm{mag}$, for the rest of the stars (BH Cep, BO Cep, VX Cas, RZ Psc) the "normal light" is represented by a broad band of data points. In most regions of the lightcurves of these stars the standard deviation of the average brightness is smaller than \pm 0.2 mag. Comparing this value with the maximum error of the eye estimates discussed in Sect. 3.2, we have to conclude that the uncertainty of the estimated magnitudes can account for a large percentage of the scatter of the data points, but short-scale activity certainly also contributes to it. Our data suggest that all of the stars, with the possible exception of RZ Psc, exhibit occasional short-lived brightenings. The existence of such brightenings has already been known from photoelectric observations so that they may not generally be attributed to plate defects.

In most parts of the lightcurves the distribution of the data points around the average is rather asymmetrical. They cover a broader magnitude interval on the lower side of the data point band. We interprete this behaviour as the consequence of minima, generally short-lived, which arise from occultations of the stars by circumstellar clouds. These so-called Algol-like minima will be studied in more detail in the following section. Here we will focus on general properties of the light variations.

We searched the data of the six stars for possible periodicities by means of the Discrete Fourier Analysis (DFA). The results are displayed in Fig. 3 as amplitude spectra.

Earlier observers of SV Cep have differentiated between medium-term wave-like light variations with durations from 100 to 1000 days and stochastic fluctuations. From a very fragmentary lightcurve we conjectured a secular decline of the stellar brightness (Friedemann et al. 1992 and references therein). The more complete data correct that picture and show that the long-term changes have a wave-like character, too. The fictitious secular decline was pretended by the highly fragmentary data. This experience underscores once again how important long and coherent time series are for the study of irregular variables. The DFA did not reveal any single dominant period. The component with the largest amplitude $(\approx 0.18 \mathrm{mag})$ has a period of $7599 \mathrm{~d}$, but several additional components with only slightly lower amplitudes are also present. Therefore, 

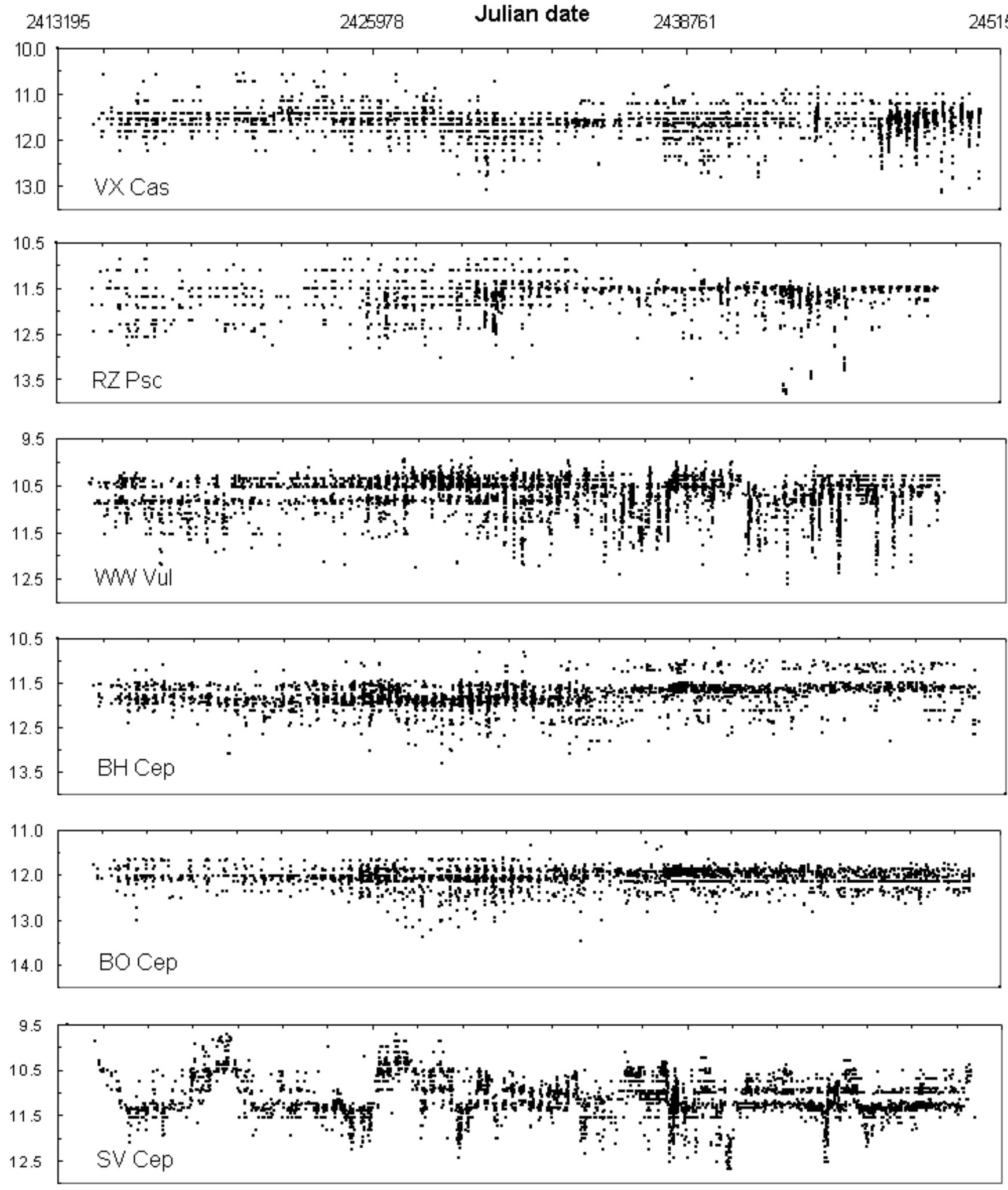

Fig. 2. Lightcurves for VX Cas, RZ Psc, WW Vul, BH Cep, BO Cep, and SV Cep based on the brightness estimates on plates of the archives at Harvard College Observatory and Sonneberg Observatory covering the period 1898-1997 and incorporating additional photometric data from the literature as described in the text. $B$ magnitudes are plotted as ordinates 

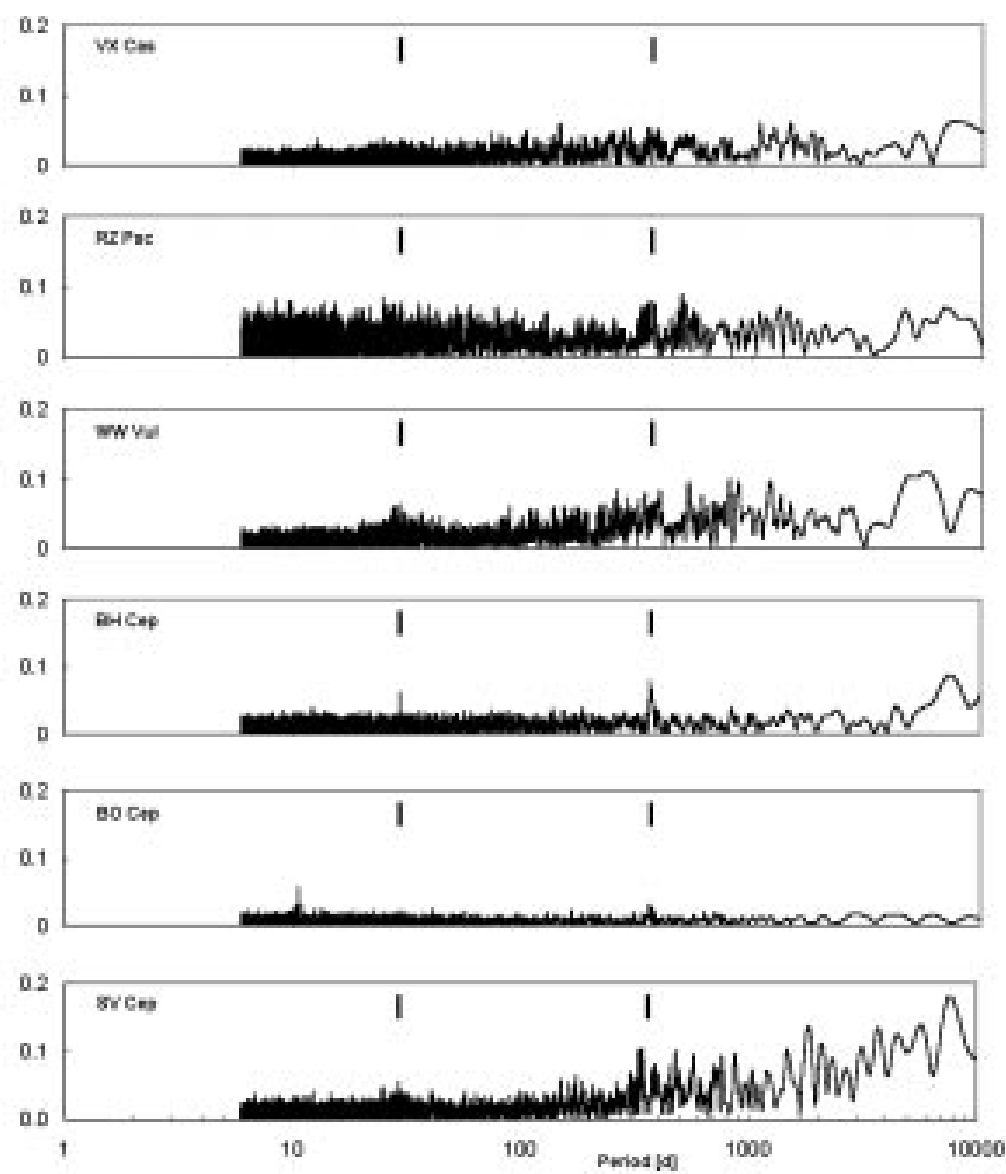

Fig. 3. Amplitude spectra for the data displayed in Fig. 2. The positions of the annual and monthly periods are indicated

we have to conclude that the wave-like light variations are largely irregular. We attribute them to intrinsic variations of the star.

The light variations of WW Vul are very similar to those of SV Cep, but the periodicities are far less marked. The component with the largest amplitude $(\approx 0.11 \mathrm{mag})$ has a period of $5714 \mathrm{~d}$, which agrees with our earlier result (Friedemann et al. 1993) satisfactorily. Again we attribute the wave-like variation to the star itself.

Our amplitude spectrum of BO Cep shows a single prominent peak corresponding to a period of $10.658289 \mathrm{~d}$. This value confirms earlier results by Grankin et al. (1991) and Wenzel (1991). Based on photoelectric photometry, these authors had also found that the amplitude of the mean lightcurve is about $0.04 \mathrm{mag}$, but minima which have amplitudes up to $0.4 \mathrm{mag}$ occasionally occur. Figure $4 \mathrm{a}$ shows the mean lightcurve based on our brightness estimates, where we computed the phase from the epoch derived by Wenzel (1991). Of course we may not expect that the regular minima with their extremely small amplitudes can be recognized in our photographic data, but obviously the occasionally occurring deeper minima have been detected in sufficient numbers to produce an accumulation of data points around the epoch of the minimum. In order to show the periodic nature of the light varia- tions more clearly, we calculated normal points and show the resulting mean lightcurve in Fig. 4b. Our amplitude of $A \approx 0.13 \mathrm{mag}$ is larger than that found by Grankin et al. (1991) and Wenzel (1991). The reason may be that the number of deep minima in our sample is larger than in their data since the distribution of the Algol-like minima shows smaller numbers during their period of observation. Moreover, our diagram reveals that sometimes very deep minima were observed. It seems remarkable that even these very deep minima occur at phase zero.

We wish to emphasize that the period of the light variations as well as the epoch hold for the full time interval covered by our data set and no glitches seem to have occurred. Because of the large standard deviation of the data points, it appears premature to discuss whether the slight asymmetry in the mean lightcurve is real or not.

The modelling of the light variations seems to call for at least two different mechanisms. The strictly periodic low-amplitude variation is very reminiscent of the lightcurve of an eclipsing binary and may be caused by an unseen secondary component in a binary system, which partially eclipses the primary every 10.658289th day. The deeper minima have to be classified as Algol-like and are presumably due to occultations by circumstellar dust clouds. To synchronize these two physically distinct 


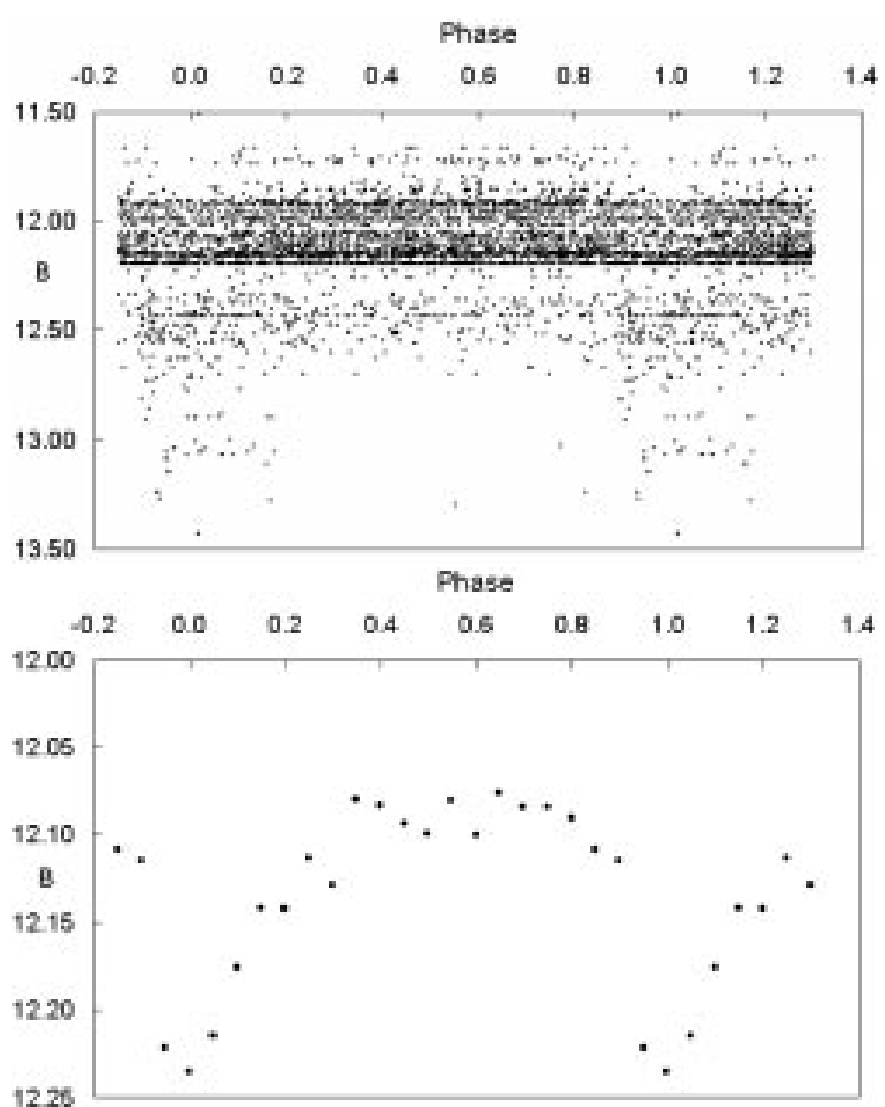

Fig. 4. Mean lightcurve of BO Cep adopting a period of $10.658289 \mathrm{~d}$. The lower diagram is based on normal points using a bin width of 0.05 . Please note that the ordinates have different scales in the diagrams

mechanisms, Grankin et al. (1991) proposed a model in which the circumstellar clouds move around the secondary but can occasionally leave it through the inner Lagrangian point onto the primary. When near the Lagrangian point, the cloud produces the observed minimum. A major objection against this model is that the dimensions of the binary are such that dust residing in a shell around the secondary is heated by the primary to temperatures which are too high for the grains to survive. Here we wish to offer an alternative mechanism. The mean lightcurve shows no indication of a secondary eclipse. We assume, therefore, that both components of the binary have identical properties and the orbital period is twice the observed period of the light variations. Adopting the mass of each star as $1.5 M_{\odot}$, the distance between the stars would be $0.2 \mathrm{AU}$. It follows from the discussion of the duration of the Algol-like minima that the clouds have distances of 2.5 AU and more from the centre of gravity of the system. Let their orbits be inclined to the orbital plane of the binary. Then an occultation will become visible from the earth whenever a cloud is near the orbital plane of the binary and in conjunction with either star. A conjunction with either star is, however, possible only if both stars are situated on, or very near the line of sight, i.e., during an eclipse. Thus a configuration where clouds which move in the orbital plane of the binary are extremely rare can account for the observed synchronization. To test this model, it is important to prove the binary nature of BO Cep and to check (e.g., by multicolour observations) whether the origin of the Algol-like minima is actually circumstellar dust clouds.

The normal light of VX Cas, BH Cep, and RZ Psc is represented by a broad band of data points without any distinct wave-like patterns. Our DFA confirms this qualitative impression. It revealed no dominant periodic component in the lightcurve of any of these three stars. This contradicts results by Shevchenko et al. (1993a,b), who claimed a period of 4.46 a for VX Cas from their photoelectric data, and by Grankin et al. (1991), who reported a period of $13.78 \mathrm{~d}$ for RZ Psc. For the latter star Zaytseva (1985) claimed a period of $12.67 \mathrm{~d}$ for minima fainter than $13^{\mathrm{m}}$. This period is also absent in our data. One has to keep in mind that the time intervals considered by these authors were much shorter than the interval covered in this study. It is therefore possible that the periods found by them are held only temporarily and cannot be recognized in the long-term data set.

To summarize the foregoing discussion of the long-term behaviour of the light variations, it must be stated that only in the case of BO Cep the existence of a stable periodic component of the lightcurve could be confirmed. The variations of the other stars are more or less irregular or cyclical at best.

\subsection{The Algol-like minima}

Short-lived minima (durations typically shorter than $10 \mathrm{~d}$ ) are a common feature of the lightcurves of all of our stars. The term Algol-like minima was applied to them because their shape can be reminiscent of the minima of eclipsing binaries. Figure 5 shows two well observed Algol-like minima as examples of both peaked and flat-bottomed minima. Although the term Algol-like minima did initially not claim to say anything about their cause, multicolour observations have accumulated convincing evidence that these minima are due to occultations of the stars by orbiting circumstellar dust clouds. However, the number of well observed minima is not very large. We will discuss the conclusions which can be drawn from the observations available in the next section. Here we will use the photographic data in an attempt to derive some statistical properties of these type of minima.

Because of the compressed timescale of Fig. 2, only the deepest Algol-like minima are clearly discernible as single observations well below the broad data bands representing the normal light of the stars. Additional minima with smaller amplitudes are present within this band as is indicated by the asymmetric distribution of the data points. Due to gaps in the time series of the photographic observations, the course of the minima is only partly covered in 
most cases. The photographic data also cannot give any hints on the cause of the minima as multicolour observation can do. Therefore, in the context of this paper the term Algol-like minimum is applied to any decrease of the stellar brightness on the timescale of days or weeks and we assume that it is caused by an occultation of the star by a circumstellar dust cloud.

Because the uncertainty of any particular data point is relatively large and the intervals between successive observations differ widely, the visual inspection of the lightcurves gives an only incomplete result and the hazard of subjective misjudgement is large. To make the search for short-lived minima as objective as possible, we applied the following procedure. First we divided the lightcurve in time intervals of equal length $\Delta t$. The mean brightness $\langle B\rangle$ and the standard deviation $\sigma$ were then calculated from the combined data of any three adjacent intervals and then taken as representative for the normal light in the middle interval. As the consequence of this procedure, the normal light of the stars was approximated by something similar to a running mean. We excluded the data points below a critical limit $B_{\text {crit }}$ in the averaging process to minimize the influence of the deep minima on the average brightness. Test runs were performed with different interval lengths $\Delta t$ to find the most appropriate parameters for each star. In the second step we selected as possible minima all observations, for which $A=B-\langle B\rangle \geq 2.5 \sigma$. If there were several observations per night, the average of them must satisfy this condition, otherwise the possible minimum was rejected. The number of minima initially selected but then rejected, may serve as one indication of how reliable the identification of a minimum really is if it is based on a single observation only. We found that the fraction of rejected minima is between 0.06 for $\mathrm{VX}$ Cas and 0.457 for SV Cep. While the fraction of rejected minima increases slightly with the total number of observations (because the number of multiple observations per night increases), the high rejection rate makes SV Cep very special. However, this is also the star with the lowest detection rate. As for Algol-like minima, this star very likely shows a very low level of activity.

Bad weather, seasonal visibility, and bright moonlight are the main causes for gaps in the time series of the observations. One problem that arises from such gaps is that we possibly cannot decide whether two successive fadings which are several days apart belong to a single minimum or represent two minima, but the star was unfortunately not observed while in its normal light. Therefore, we have defined that successive fadings belong to a common minimum when they occurred not more than $10 \mathrm{~d}$ apart. Lack of observations is also the reason why only upper limits for the duration of the minima could often be determined or why the duration could not be derived at all. A third consequence of the gaps is that a certain number of Algollike minima remains undetected because the most are of short duration only.

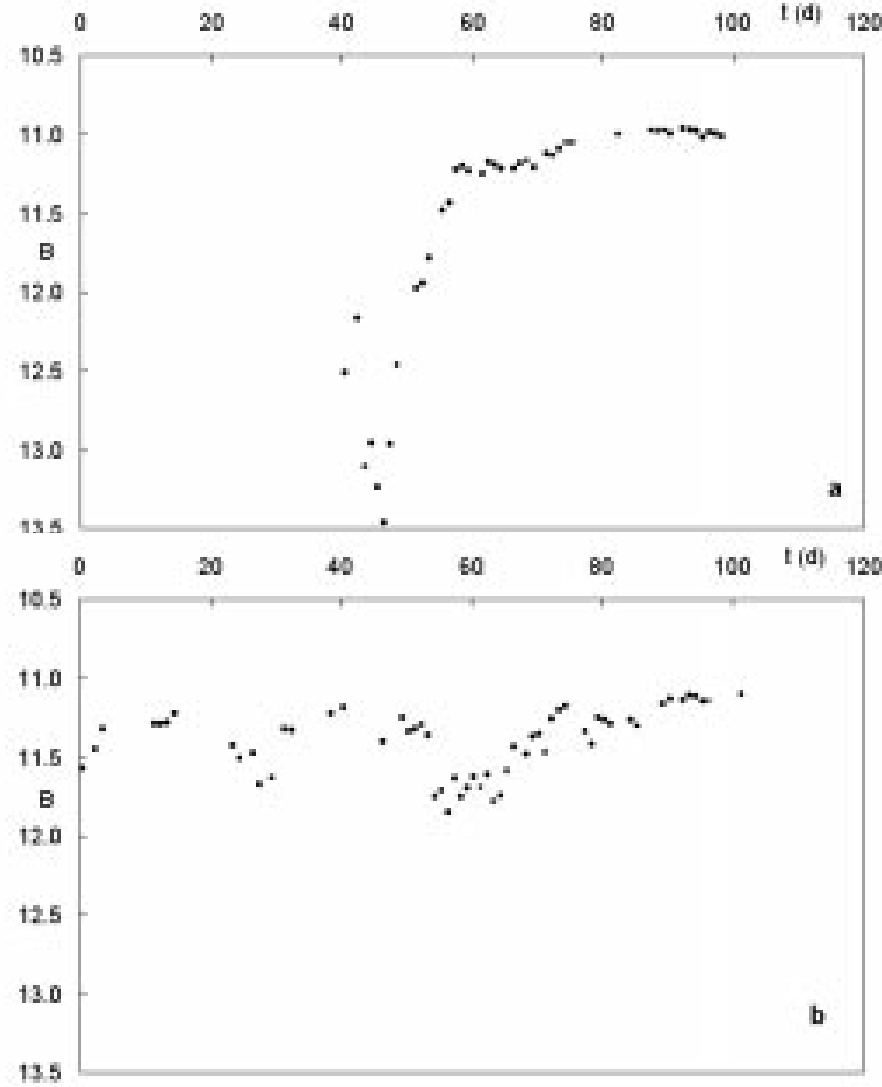

Fig. 5. Examples of a deep peaked Algol-like minimum a) and a trough-like minimum b). Photoelectric data for VX Cas by courtesy of Shevchenko (private communication)

Table 4 summarizes some general properties of the Algol-like minima accepted as such by our standards. In addition to the total number of minima found for each star, we listed the number of minima, for which durations $\tau$ could be derived, the mean amplitude of the 5 deepest minima, the mean duration of the minima with durations $\leq 4 \mathrm{~d}$, the parameters of the minimum search procedure, and the mean number of observations per minimum.

To get some idea of the reliability of the numbers, we first look for evidence of incompleteness. As already mentioned above and also discussed in same detail by Friedemann et al. (1995), gaps in the observational data have prevented us from detecting all minima. We would expect, however, that the gaps become shorter and the number of detections increases with an increasing number of observations. If the stars are compared with each other, no close correlation between the total number of observations and the total number of minima detected exists. This means that the frequency of the minima varies among our stars. A trend is, however, visible if for each star the number of minima per year is plotted against the number of observations per year. It appears that a large number of observations are a necessary condition for a large detection rate of minima, but it is not sufficient. On the contrary, a low number of observations always resulted 
Table 4. Characteristics of Algol-like minima

\begin{tabular}{lcccccc}
\hline & VX Cas & RZ Psc & WW Vul & BH Cep & BO Cep & SV Cep \\
\hline $\begin{array}{l}\text { Total number of observations } \\
\text { Total number of minima }\end{array} n_{\text {min }}$ & 3907 & 2971 & 6958 & 3976 & 3679 & 6000 \\
$\begin{array}{l}\text { Number of minima } \\
\text { with the duration estimates }\end{array}$ & 107 & 93 & 213 & 183 & 95 & 44 \\
$\begin{array}{l}\text { Mean amplitude } \bar{A} \text { of the } \\
\text { deepest minima (mag) }\end{array}$ & 56 & 46 & 141 & 73 & 53 & 25 \\
$\begin{array}{l}\text { Mean duration of minima } \\
\text { with } \tau \leq 4 \mathrm{~d}(\mathrm{~d})\end{array}$ & 1.28 & 1.65 & 1.33 & 1.29 & 1.15 & 1.16 \\
$\begin{array}{l}\text { Bin width } \Delta t \text { in calculating } \\
\text { the running mean }\langle B\rangle(\mathrm{d})\end{array}$ & 3.1 & 2.4 & 2.9 & 2.9 & 3.5 & 3.2 \\
$n_{\text {tot }} / n_{\text {min }}$ & 240 & 240 & 120 & 240 & 240 & $60 ; 80 ; 120$ \\
\hline
\end{tabular}

in the detection of few minima. With other words, for a certain number of observations, there is an upper limit for the number of minima detected.

It is clear that a large scatter of the data points of the normal light may mask some minima, especially those with relatively low amplitudes. The relatively large error produces spurious fadings. It follows from our selection criterion for Algol-like fadings $(A \geq 2.5 \sigma)$ that the probability of a selected fading to be due to the estimation error is about 0.0062 . For all stars but SV Cep the number of minima found is much larger than predicted from the scatter of the data points alone. Therefore, most of these minima should be real and suited for further analysis. Because of the strong wave-like variability of SV Cep, the portions of the lightcurve where $\sigma$ is relatively small are much shorter than for the lightcurves of the other stars. The recognition of Algol-like minima, especially minima with small amplitudes, is hindered considerably. The number of fadings initially detected (81) is only twice the number expected based on the scatter of the data (37). From this and the high rejection rate we conclude that the fraction of the genuine minima among the total number of accepted minima is smaller for SV Cep than for the other stars.

The standard deviation $\sigma$ computed in the averaging procedure is not constant over the whole lightcurve. Consequently we could not select the minima everywhere from the same minimum amplitude upward and the numbers of small-amplitude minima are systematically too low for $A<2.5 \max (\sigma)$. We made an attempt to correct crudely for this systematic effect. We assume that both the number of minima and the relative distribution of their amplitudes are constant over the whole time interval. Then the number of minima with $A \leq 2.5 \sigma$ is too low by the factor $\int_{0}^{\sigma} n\left(\sigma^{\prime}\right) \mathrm{d} \sigma^{\prime} / n_{\min }$, where $n(\sigma)$ and $n_{\text {min }}$ are the number of minima detected in parts where the observations have a standard deviation of $\sigma$ and the total number of minima, resp. Figure 6 shows the normalized distributions of the amplitudes of the Algol-like minima for our six stars. Both the uncorrected and corrected distributions were plotted. While the uncorrected distributions apparently signalize significant differences among the stars, the serious influence of the different values of $\sigma$ becomes evident through our correction procedure. Apart from RZ Psc the amplitude distributions seem to be quite similar with about 50 per cent of the minima having amplitudes smaller than 0.5 mag. In the case of RZ Psc the low number of shallow minima appears to be not an effect of observational errors alone but a property of the circumstellar shell.

In Fig. 7 we present the relative distributions of the duration of the minima. The number of minima is not corrected for any incompleteness due to the amplitudeto- $\sigma$ ratio. Different distributions occur among stars with similar time series. A comparison with the data in Table 3 shows that a large number of observations does not necessarily mean a higher fraction of shortest minima. Therefore, we feel that the low rate of short minima in the lightcurve of VX Cas may not be attributed to a lack of observations alone. On the contrary, SV Cep and RZ Psc have exceptionally large fractions of short minima. However, SV Cep is not a fully convincing case. Because for this star the fraction of spurious minima may be larger than for the other stars, the distribution function could be biased. More interesting is the preponderance of short minima in the case of RZ Psc. Since this star is physically different (it is of relatively late spectral type), the different distribution functions for amplitude and duration may well point to differences in the shell properties between young stars of early and late spectral types.

\subsection{Photometric properties of the Algol-like minima - $R$} values and colour excess ratios

As discussed in some detail by Friedemann et al. (1993, 1994a), multicolour photoelectric observations of Algollike minima make it possible to determine the extinction law of the circumstellar dust and derive the extinction properties of the grains. For four (WW Vul, SV Cep, VX 


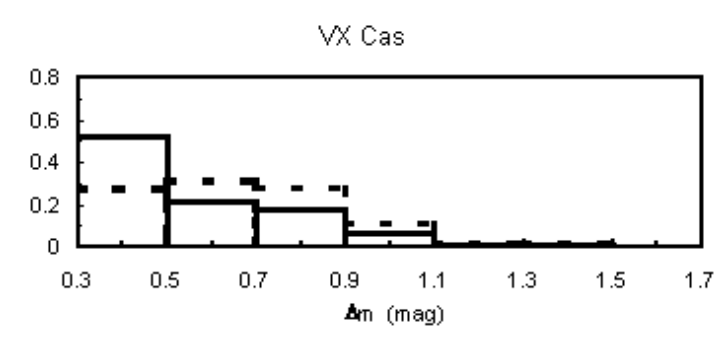

RZ Psc

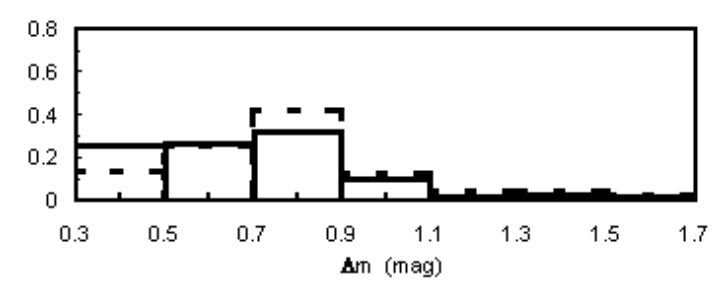

WW Vul

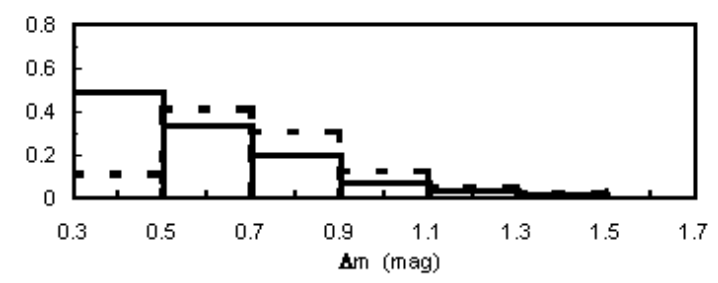

BH Cep

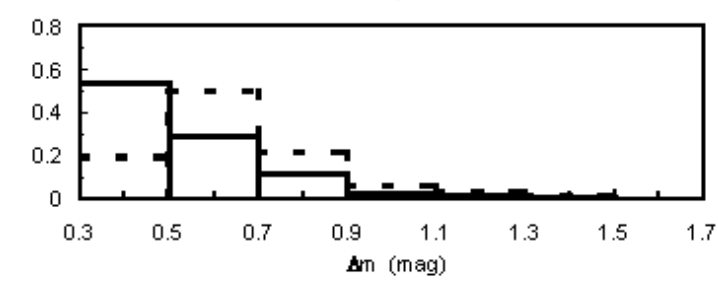

BO Cep

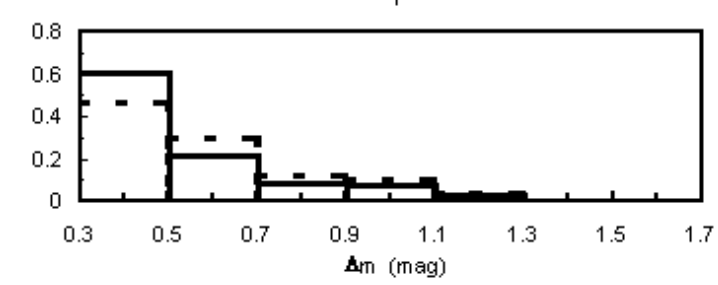

SVCep

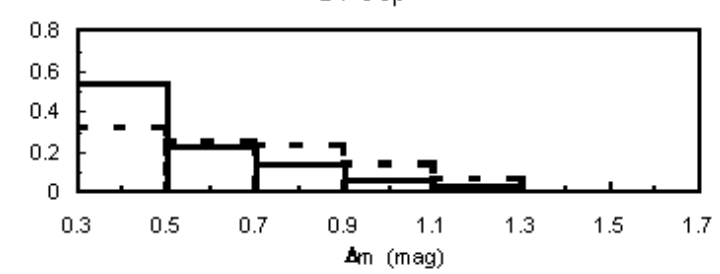

Fig. 6. Relative frequency distribution of the amplitudes of the Algol-like minima. Both the observed distribution (broken line) and the distribution corrected for the effects of the varying standard deviation over the lightcurves (solid line) are shown
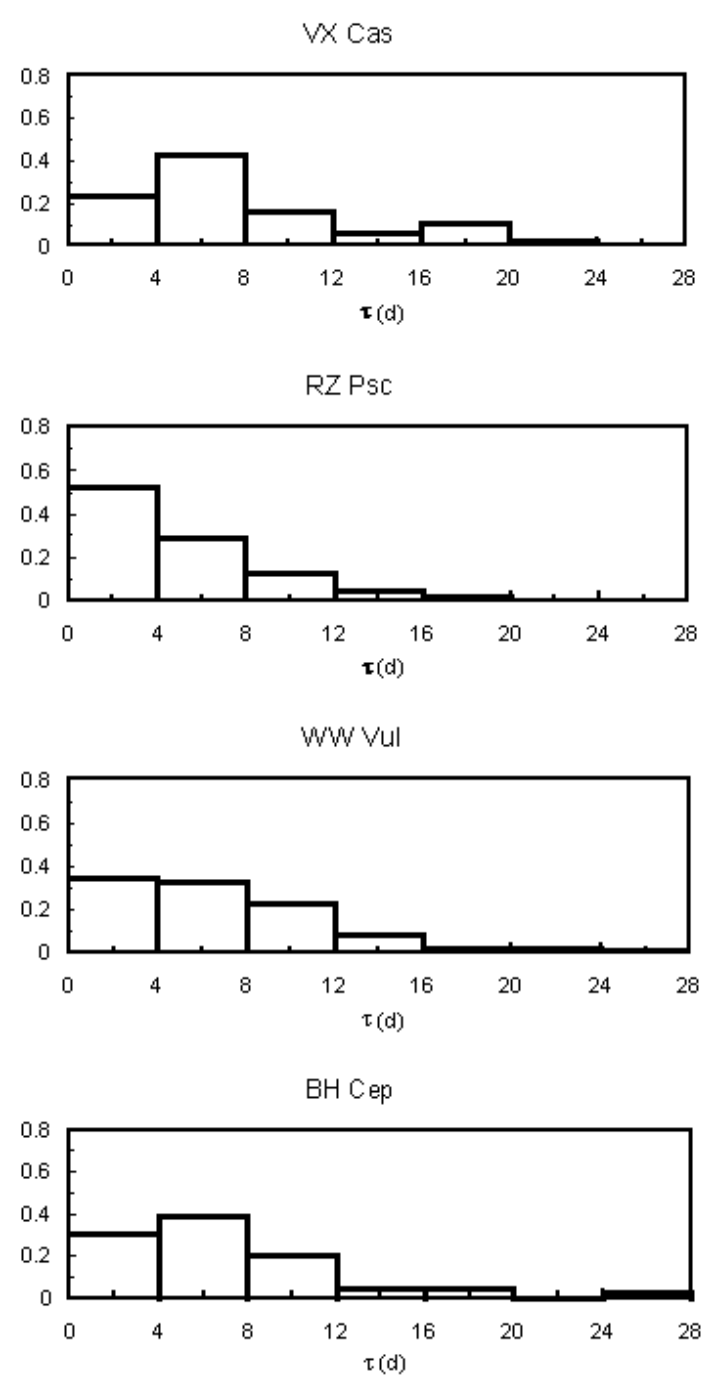

BO Cep
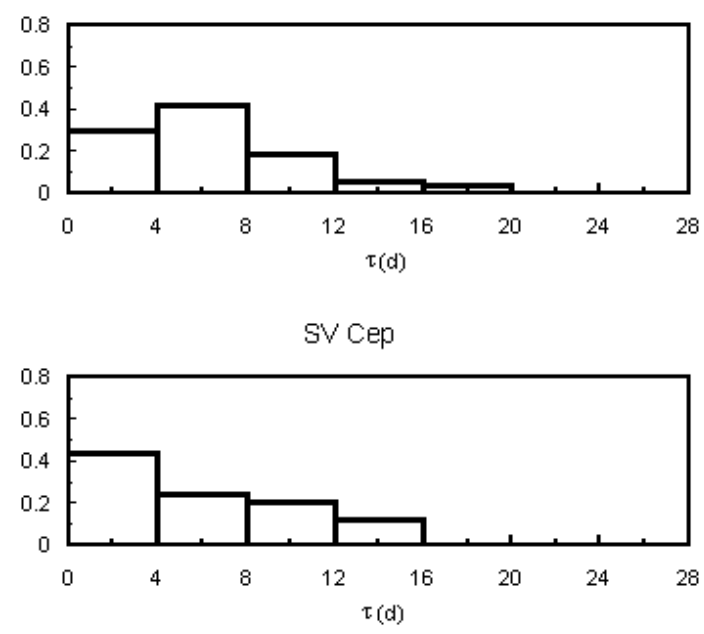

Fig. 7. Relative frequency distribution of the durations of the minima 


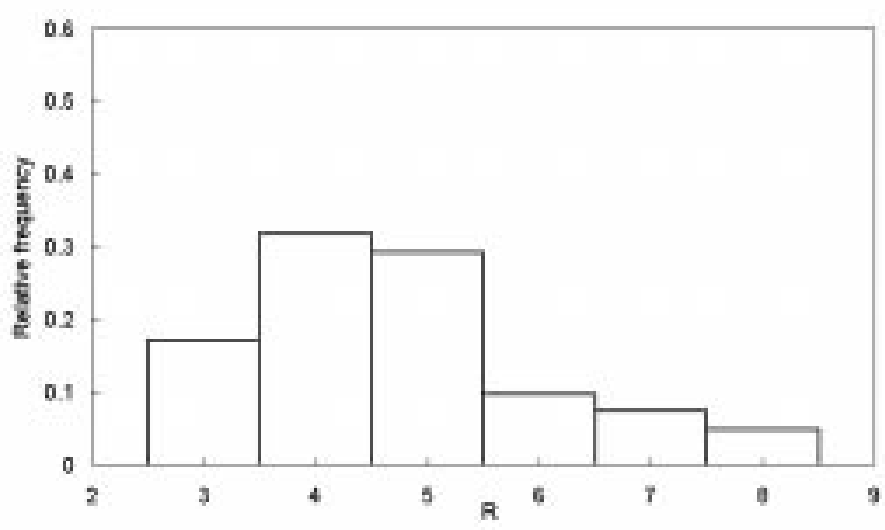

Fig. 8. Relative frequency distribution of the $R$ values of 41 Algol-like minima observed for WW Vul, SV Cep, VX Cas, and RZ Psc

Cas, and RZ Psc) out of the six stars dealt with here, photoelectric $U B V R$ and $U B V$ photometry was available from several authors (see Sect. 4.1. for references). These data were used to investigate the photometric properties of a total of 41 Algol-like minima (17 of WW Vul, 3 of SV Cep, 11 of VX Cas and 10 of RZ Psc) by means of colour-magnitude and two-colour diagrams.

As shown by Friedemann et al. (1993, 1994a), the path that the star follows in the colour-magnitude diagram during an Algol-like minimum is distinctly different from the path that a change of the intrinsic stellar parameters, such as luminosity or effective temperature, would produce and may be interpreted as a reddening line. Different minima often have reddening lines with different values of $R=A_{V} / E_{B-V}$. Also from the two-colour diagrams it is clearly seen that during a minimum the colours change according to reddening lines, but that different slopes $E_{U-B} / E_{B-V}$ and $E_{V-R} / E_{B-V}$ apply to different minima.

We calculated the $R$ values and colour excess ratios from linear regressions for all 41 minima. Possible errors of both variables were taken into account. One standard deviation is typically 0.15 mag for the colour excess ratios and 0.3 for $R$. In Fig. 8 we show the relative frequency distribution of the $R$ values of our sample. Values larger than that typical of the diffuse interstellar medium are frequent. The mean circumstellar value is $\bar{R}=4.4 \pm 0.2$. The relatively wide scatter in the $R$ values implies that the different minima were caused by dust clouds consisting of grain populations with quite different extinction properties.

No photoelectric observations of deep Algol-like minima are available for $\mathrm{BH}$ and $\mathrm{BO}$ Cep. However, Pugach (1988) concluded from his $U B V R$ observations of BH Cep that the small-amplitude variations are compatible with $R=3.2$ and the circumstellar and interstellar dust may, therefore, be similar.

To check whether the $R$ values derived by us are compatible with the common assumption that larger $R$ values

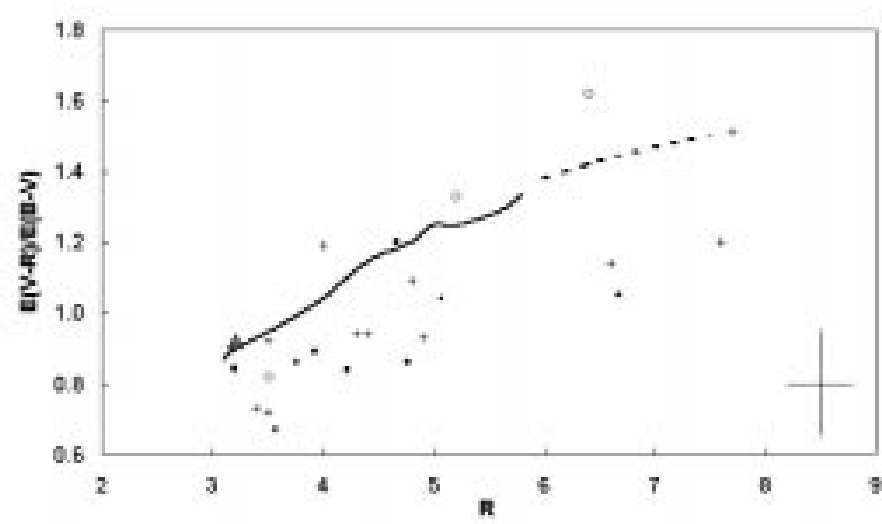

Fig. 9. Relation between reddening parameter $R$ and colour excess ratio $E(V-R) / E(B-V)$ for WW Vul (crosses) SV Cep (open diamonds) and VX Cas (filled circles). The filled square is the result by Pugach (1988) for BH Cep. The grey triangle marks the standard value of the interstellar mean. The solid line (and the dashed part of it as an extrapolation from us) represent model calculations by Steenman \& Thé (1991) discussing anomalous extinction curves. The error bars in the lower right corner indicate one typical standard deviation

are the consequence of larger grain sizes, we compared our observational results with model calculations by Steenman \& Thé (1991). They assumed the dust model of Mathis et al. (1977) for the general interstellar medium and studied the effect of changing the lower and/or upper limits of the particle size distribution on the reddening law. The solid line in Fig. 9 shows the predicted effect of increasing the minimum particle size. The dashed line is our extension of this model to higher $R$ values. Within the observational errors the observed colour excess ratios and $R$ values agree well with the model predictions. This seems to be convincing evidence for the assumption that the individual clouds responsible for the various Algol-like minima may be characterized by different depletion of small grains. The small systematic difference between the theoretical curve and the majority of the observations may indicate that the circumstellar reddening law is not altogether identical to the interstellar one.

\section{Infrared radiation}

The IRAS satellite detected infrared radiation from all stars of our sample with the exception of RZ Psc. This radiation is unquestionably thermal emission from circumstellar dust and opens a way to estimate the total mass of circumstellar dust by modelling the spectral energy distribution.

We tried to reproduce the observed spectral energy distributions in the infrared by simple spherically symmetric models. The shell is defined by the inner and outer radii of the shell, $r_{\mathrm{i}}$ and $r_{\mathrm{o}}$, and the density within it is approximated by a power law $\rho(r) \propto r^{-d}$. The nature and optical properties of the dust grains are not known very 
well. We assume the dust to be a mixture of bare silicate and graphite spheres with the optical constants as given by Draine (1985) and a size distribution $n(a) \propto a^{-3.7}$, resembling the distribution function of Mathis et al. (1977). The stellar radiation is approximated by a black body of the effective temperature of the star.

The relatively small optical depth of the shell is an a-posteriori justification for assuming a smooth density distribution in the model calculations. In the infrared we observe the emission from the whole shell, and since the average optical depth is small, we can expect that geometrical effects due to the cloudy structure are rather small. A related argument can be used to justify the assumption of spherical symmetry. There is growing evidence that circumstellar matter also around Herbig Ae/Be stars is distributed in the form of disks (Waters \& Waelkens 1998). However, as long as the matter is optically thin, the actual geometry does not really matter because the differing geometries can be accounted for by changing the exponent $d$ of the density distribution. For instance, an exponent $d$ found by fitting a spherically symmetric model is equivalent to an exponent $d_{\text {disk }}=d-1$ of a disk of constant width.

Table 5 lists parameters of our best spherically symmetric models. For BO Cep we also give the parameters of the model that is based on the binary hypothesis outlined in Sect. 4.2. Figure 10 shows the comparison of the models for VX Cas, BH Cep, and BO Cep with the observations. For similar comparisons for WW Vul, RZ Psc, and SV Cep see Friedemann et al. (1994a).

In an earlier paper we argued that the density distribution may be reflected in the minimum duration distribution if the circumstellar matter is primarily concentrated in the obscuring clouds (Friedemann et al. 1995). The duration of a minimum depends on the orbital velocity of the cloud and, therefore, on its distance from the star. If we approximate distribution of the minimum durations $\tau$, $N(\tau)$, by

$N(\tau) \propto \tau^{-\alpha}$,

then the number of clouds per unit volume, $n_{\mathrm{c}}(r)$, should follow the power law

$n_{\mathrm{c}}(r) \propto r^{-\left(\frac{\alpha}{2}+1\right)}$.

Denoting the mean dust mass of a cloud at distance $r$ by $\bar{m}_{\mathrm{c}}(r)$, the radial density distribution of the dusty matter concentrated in the clouds is

$\rho_{\mathrm{c}}(r) \propto \bar{m}_{\mathrm{c}}(r) \cdot r^{-\left(\frac{\alpha}{2}+1\right)}$.

From the modelling of the infrared excess by means of spherically symmetric dust shells, we most often found an exponent of $d \approx 1.5$ for the density distribution (see Table 5), implying a power law for the minimum duration distribution with $\alpha \approx 1$, if most of the circumstellar dust is concentrated in clouds and the dust masses within the clouds are independent of their distance from the star. A look at Fig. 7 shows that a power law is rather a bad
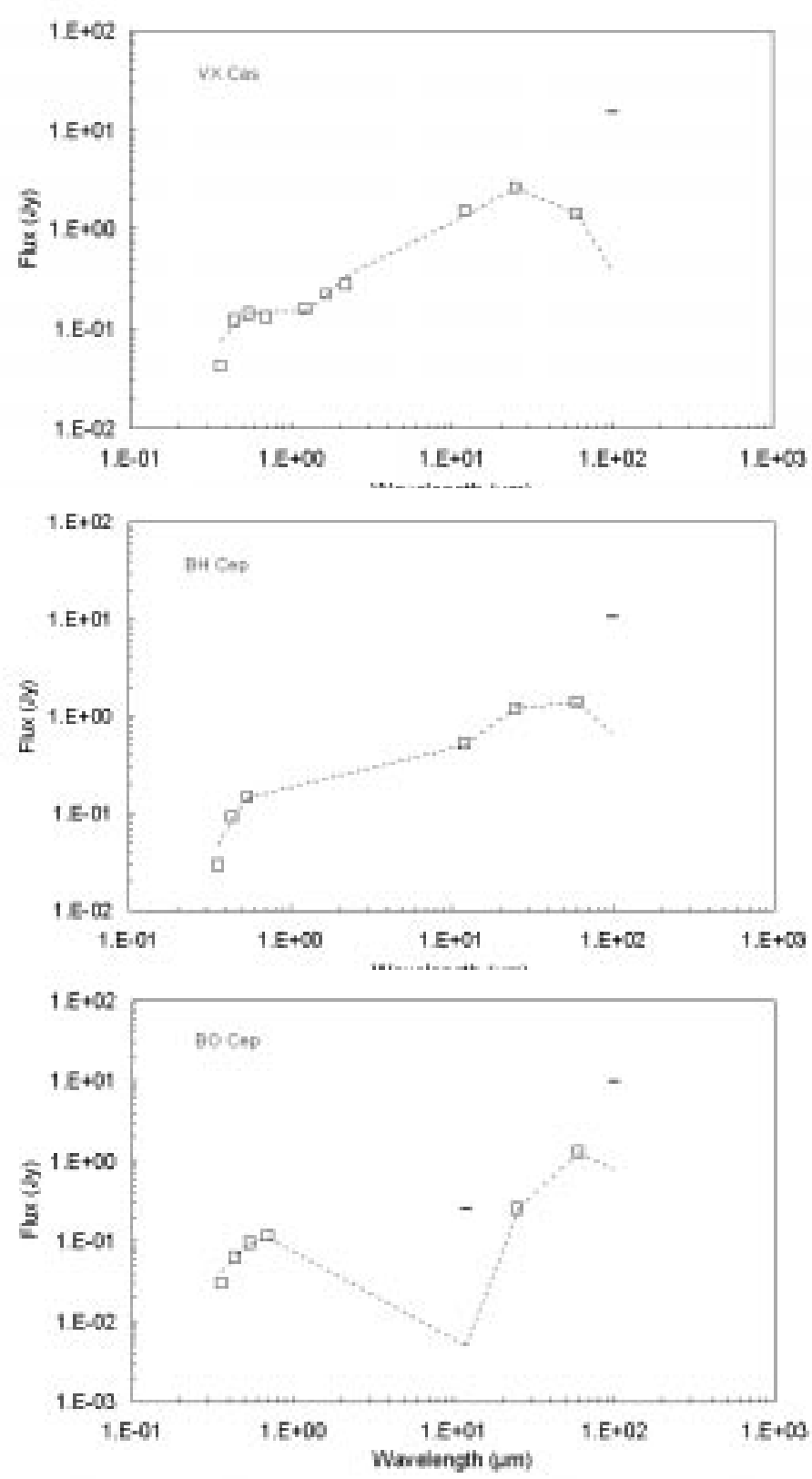

Fig. 10. Comparison of the observed spectral energy distributions with the model fits. Bars denote upper limits

representation of the distribution function. With the possible exception of SV Cep and RZ Psc, it would predict much more minima of short duration than observed. As we have mentioned above, we could have underestimated the number of shorter minima systematically. Because of gaps in the time series we cannot detect a certain fraction of the shortest minima and we cannot decide whether the data points belong to, say, two short minima or a longer one. If we take the duration distributions at face value, two possible explanations come to mind. First, the dust masses of the innermost clouds could be systematically larger than the masses of the clouds lying farther out. While a larger mass make the cloud more stable against 
Table 5. Characteristics of the best fitting models

\begin{tabular}{lcccccc}
\hline & VX Cas & RZ Psc $^{a}$ & WW Vul & BH Cep & BO Cep $^{b}$ & SV Cep \\
\hline Star: & 10000 & 5250 & 9520 & 6400 & 6900 & 10500 \\
$T_{\text {eff }}(\mathrm{K})$ & 53.8 & 5.5 & 54.0 & 7.4 & $3.85(7.7)$ & 93.8 \\
Luminosity $L\left(L_{\odot}\right)$ & 850 & 480 & 550 & 400 & $400(566)$ & 530 \\
$\begin{array}{l}\text { Distance } D(\mathrm{pc}) \\
\text { Circumstellar shell: }\end{array}$ & 1.67 & $(4.0)$ & 2.7 & 11.7 & $250.7(355)$ & 13.4 \\
$\begin{array}{l}\text { Inner boundary } r_{\mathrm{i}}(\mathrm{AU}) \\
\text { Outer boundary } r_{\mathrm{o}}(\mathrm{AU})\end{array}$ & $8.4210^{2}$ & $\left(4.410^{3}\right)$ & $4.710^{3}$ & $2.4810^{3}$ & $2.9310^{3}\left(4.1410^{3}\right)$ & $5.910^{3}$ \\
$\begin{array}{l}\text { Exponent of } \\
\text { density distribution } d\end{array}$ & 1.0 & $(1.5)$ & 1.45 & 1.35 & & 1.5 \\
$\begin{array}{l}\text { Total mass } M\left(M_{\odot}\right) \\
\text { Lower limit of } \\
\text { minima duration } \tau_{\min }(\mathrm{d})\end{array}$ & $2.910^{-4}$ & $\left(3.510^{-4}\right)$ & $6.310^{-4}$ & $6.210^{-4}$ & $1.210^{-3}\left(2.410^{-3}\right)$ & $7.310^{-4}$ \\
\hline
\end{tabular}

${ }^{a}$ RZ Psc was not detected by IRAS. The values adopted in the model only illustrate that plausible numbers are in accordance with the nondetection.

${ }^{b}$ The values in brackets are for the hypothetical central binary of 2 identical F2 stars.

tidal disruption and enlarges its lifetime, the extinction by the cloud and thus the depth of the minimum would also increase. However, there is no indication in the data that the shortest minima have larger amplitudes than the longer ones. Second, the proportion of dust that is not concentrated in clouds but diffusely distributed through the circumstellar shell could be larger in the innermost region of the shell, e.g., as a consequence of a greater instability of the clouds near the star. Here we would like to mention the work by Krivova (1997) on WW Vul, who constructed a model where the infrared radiation is primarily emitted by a diffusely distributed shell with some absorbing clouds producing the Algol-like minima.

The inner boundary $r_{\mathrm{i}}$ of the circumstellar shells and the duration of the shortest minima $\tau_{\text {min }}$ offer a further connection between the models based on the infrared emission and the properties derived for the cloud ensembles. Assuming circular orbits, the duration of a central occultation by a cloud at distance $r$ from the star is $\tau(r)=4 R_{*} \sqrt{r / G M_{*}}$,

where $R_{*}$ and $M_{*}$ are the radius and the mass of the star, respectively, and the cloud radius is assumed to be equal $R_{*}$. The shortest minima should occur for $r=r_{\mathrm{i}}$. The resulting values $\tau_{\min }$ are listed in the last line of Table 5 . Stellar masses and radii are adopted as appropriate for main sequence stars of the respective spectral class.

Comparing the lower limits $\tau_{\min }$ in Table 5 with the histograms of Fig. 7, a large discrepancy occurs in the case of BO Cep. There are smaller differences for BH and SV Cep, too, but we do not think that they are serious enough to argue against the basic assumption, i.e., that cloud ensemble and infrared emitting circumstellar dust shell occupy the same space and are identical. Obviously, this basic assumption has to be abandoned for BO Cep. The infrared emitting shell is located much farther from the star than the objects responsible for the minima. It remains an open question whether this spatial separation is a key to understand the synchronization between the periodic component of the light variations and occasionally occurring deeper minima.

\section{Conclusions}

For the six young stars VX Cas, BH Cep, BO Cep, SV Cep, RZ Psc, and WW Vul we have presented photometric data covering a period of about one century. Most of the data consist of brightness estimates on photographic plates from the collections of Harvard and Sonneberg observatories. Additional data come from both published and unpublished multicolour photoelectric observations as well as from visual brightness estimates collected by AAVSO. All the stars are variables of the UX Ori type.

These comprehensive data sets were used to make a comparative study of the light variations of the stars. The major results can be summarized as follows:

1. The light variations besides the Algol-like minima are different among the stars and vary from a pronounced wave-like pattern (SV Cep) to a nearly constant normal light (BO Cep);

2. With the exception of BO Cep, no period could be found in the light variations;

3. The period found for BO Cep confirms an earlier finding by Wenzel (1991). The orbital period of the binary is synchronized with the occurrence of Algol-like minima;

4. Algol-like minima were observed with all stars. Their number varies among the stars and during the time intervals covered by the observations;

5. The amplitudes and durations of the Algol-like minima agree with the concept of a cloudy circumstellar shell;

6. Multicolour observations of individual minima of VX Cas, SV Cep, WW Vul, and RZ Psc prove dust 
extinction as cause of the Algol-like minima. The reddening law points to larger grain radii than in interstellar space;

7. Modelling the infrared excess by spherically symmetric dust shells shows that the density distribution follows a power law with a exponent of -1.0 to -1.6 ;

8. Apart from BO Cep, infrared observations call for circumstellar shells that are generally compatible with the properties derived from the light variations.

Acknowledgements. The authors are indebted to Dr. I. Shapiro, director of Harvard College Observatory, for the permission to use the plate collection. Three of us (C.F., J.G., H.-G.R.) thank Dr. M. Hazen, curator of the plate collection, for her kind support and hospitality during their stays. We wish also to thank Dr. C. la Dous, director of Sonneberg Observatory, for the permission to use the plate collection. In this research, we have used, and acknowledge with thanks, data from the AAVSO International Database, based on observations submitted to the AASVO by variable star observers world-wide. Furthermore, we would like to thank Dr. V.S. Shevchenko for providing us with his extensive $U B V R$ data set of VX Cas. Finally, we thank our referee, J. Lub, for his constructive remarks that helped to improve the presentation of this paper. The work was partially supported by Deutsche Forschungsgemeinschaft (Fr 963/3-1) and the German Bundesministerium für Bildung, Wissenschaft, Forschung und Technologie (Förderkennzeichen 05 3JN13A).

\section{References}

Draine B.T., 1985, ApJS 57, 587

Friedemann C., Reimann H.-G., Gürtler J., 1992, A\&A 255, 246

Friedemann C., Reimann H.-G., Gürtler J., Tóth V., 1993, A\&A

Friedemann C., Reimann H.-G., Gürtler J., 1994a, Ap\&SS 212, 221277,184

Friedemann C., Hoffrichter, J., Reimann, H.-G., Gürtler J., 1994b, Mitt. Veränderliche Sterne, Sonneberg 12, 188

Friedemann C., Gürtler J., Reimann H.-G., 1995, A\&A 300 , 269
Friedemann C., Reimann H.-G., Gürtler J., 1996, A\&AS 120, 429

Grankin K.N., Ibragimov M.A., Melnikov S.Yu., et al., 1991, Inf. Bull. Variable Stars, No. 3626

Herbig G.H., Bell K.R., 1988, Lick Obs. Bull. No. 1117

Home J., Friedemann C., Reimann H.-G., 1994, Mitt. Veränderliche Sterne, Sonneberg 12, 199

Kardopolov V.I., Filip'ev G.K., 1981, Circ. Astron. Inst. Uzbekistan Acad. Sci. 96, 34

Kardopolov V.I., Filip'ev G.K., 1985a, Perem. Zvezd. 22, 103

Kardopolov V.I., Filip'ev G.K., 1985b, Perem. Zvezd. 22, 153

Kardopolov V.I., Shutiomova N.A., 1980, Perem. Zvezd. 21, 305

Kardopolov V.I., Sahanionok V.V., Shutiomova N.A., 1980, Perem. Zvezd. 21, 310

Kholopov P.N., 1985, General Catalogue of Variable Stars, 4th ed., Moscow, Nauka

Krivova N.A., 1997, Astron. Lett. 23, 327

Mathis J.S., Rumpl W., Nordsieck K.H., 1977, ApJ 217, 425

Pugach A.F., 1979, Astrometr. Astrofiz. 39, 8

Pugach A.F., 1988, Inf. Bull. Variable Stars, No. 3181

Pugach A.F., Kovalchuk G.U., 1983, Perem. Zvezd. 22, 9

Reimann H.-G., Friedemann C., 1991, Inf. Bull. Variable Stars, No. 3668

Reimann H.-G., Gürtler J., Friedemann C., Käufl H.U., 1997, A\&A 326, 271

Shevchenko V.S., 1998 (private communication)

Shevchenko V.S., Grankin K.N., Ibragimov M.A., Melnikov, S.Yu., Yakubov S.D., 1993a, Ap\&SS 202, 121

Shevchenko V.S., Grankin K.N., Ibragimov M.A., Melnikov, S.Yu., Yakubov S.D., 1993b, Ap\&SS 202, 137

Steenman H., Thé P.S., 1991, Ap\&SS 184, 9

Thé P.S., de Winter D., Perez M.R., 1994, A\&AS 104, 315

Waters L.B.F.M., Waelkens C., 1998, ARA\&A 36, 233

Wenzel W., 1969, Mitt. Veränderliche Sterne, Sonneberg 5, 75

Wenzel W., 1991, Inf. Bull. Variable Stars, No. 3647

Wenzel W., Brückner V., 1978, Mitt. Veränderliche Sterne, Sonneberg 8, 35

Zaytseva G.V., Lyutyi V.M., 1997, Astr. Lett. 23, 242

Zaytseva G.V., 1985, Perem. Zvezd. 22, 181

Zaytseva G.V., 1970, Perem. Zvezd. 17, 294

Zhelezniakova A.I., Kardopolov V.I., 1980, Perem. Zvezd. 21, 301 